\title{
ASSESSING A LINEAR NANOSYSTEM'S LIMITING RELIABILITY FROM ITS COMPONENTS
}

\author{
NADER EBRAHIMI,* Northern Illinois University
}

\begin{abstract}
Nanosystems are devices that are in the size range of a billionth of a meter $\left(1 \times 10^{-9}\right)$ and therefore are built necessarily from individual atoms. The one-dimensional nanosystems or linear nanosystems cover all the nanosized systems which possess one dimension that exceeds the other two dimensions, i.e. extension over one dimension is predominant over the other two dimensions. Here only two of the dimensions have to be on the nanoscale (less than 100 nanometers). In this paper we consider the structural relationship between a linear nanosystem and its atoms acting as components of the nanosystem. Using such information, we then assess the nanosystem's limiting reliability which is, of course, probabilistic in nature. We consider the linear nanosystem at a fixed moment of time, say the present moment, and we assume that the present state of the linear nanosystem depends only on the present states of its atoms.
\end{abstract}

Keywords: Binomial distribution; linear nanosystem; reliability; associated random variables

2000 Mathematics Subject Classification: Primary 60N05

Secondary 60G55; 90B25

\section{Introduction}

Over the past several years, nanoscience and nanotechnology have become two of the most important fields at the forefront of physics, chemistry, engineering, and biology. Nanoscience is the study of the world on the nanometer scale, from approximately one nanometer to several hundred nanometers. Nanoscience occurs at the intersection of traditional science and engineering, quantum mechanics, and the most basic processes of life itself. Nanotechnology, on the other hand, encompasses how we harness our knowledge of nanoscience to manipulate atoms to fabricate nanodevices. Manufacturing, also referred to as 'bottom-up nanostructure preparation', is based on building nanodevices by putting atoms together with chemical bonding or through other means. Properties of manufactured nanosystems depend on how atoms are rearranged, and their reliabilities will be limited by the strengths of the bonds holding them together. We refer the reader to Drexler (1992), Ratner and Ratner (2003), Poole and Owens (2003), Bahar et al. (2005), Bhushan (2007), Tabata and Tsuchiya (2008), and the references therein for an introduction and advances in both fields.

In the fields of nanoscience and nanotechnology, much attention has been given to the dual problem of designing nanosystems with novel physical properties and how such systems can be fabricated. Receiving less attention has been the question of nanosystem reliability: how does a nanosystem fail and how long does a nanosystem survive under typical operation conditions?

Received 28 February 2008; revision received 20 June 2008.

* Postal address: Division of Statistics, Northern Illinois University, DeKalb, IL 60115, USA.

Email address: nader@math.niu.edu 
Today, high reliability is necessary to guarantee the advancement and utilization of nanosystems due to the fact that they account for a high proportion of costs of newly designed multiscale systems.

Our goal in this paper is to develop general statistical methodologies for assessing the reliability of linear nanosystems. Our analysis uses a probability modeling formulation that exploits the internal structure of a linear nanosystem. Specifically, we consider a linear nanosystem consisting of $N$ atoms $A=\{1,2, \ldots, N\}$ acting as components of the system. Usually, for a linear nanosystem, $N$ is very large $(N \rightarrow \infty)$. The formation of a linear nanosystem essentially involves putting its atoms where they should be. The system can fail because its atoms rearrange. To indicate the state of each atom $i$, the binary indicator variable $X_{i}$ is defined by

$$
X_{i}= \begin{cases}1 & \text { if atom } i \text { is displaced } \\ 0 & \text { if atom } i \text { is not displaced }\end{cases}
$$

for $i=1, \ldots, N$. Usually, displacement occurs owing to one atom being either attracted or repelled by another atom. It should be emphasized that, for a linear nanosystem, atoms can move in one direction only. Without loss of generality, we assume that the atoms can move in the $x$-direction only. Examples of such nanosystems are solid nanofibers, nanowires, nanorods, and nanotubes.

We should note that in the conventional reliability theory $X_{i}$ is defined to be 1 if the component is functioning and 0 if the component fails; see Barlow and Proschan (1981) and Ebrahimi (1990). Also, see Natvig (2007), which covers multistate reliability theory. For a linear nanosystem, however, defining $X_{i}$ in this way does not make sense because elements chosen to create linear nanosystems are usually those with stable nuclei and, therefore, in principle, they are ageless.

In order to explore complex partial patterns of atoms in three dimensions, we represent (1) in the following way. Suppose that $X_{i j k}$ is the state of the atom located at the point $(i, j, k)$ in $D_{N_{1}, N_{2}, N_{3}}$, where $D_{N_{1}, N_{2}, N_{3}}=\left\{\left(i_{1}, i_{2}, i_{3}\right): 1 \leq i_{1} \leq N_{1}, 1 \leq i_{2} \leq N_{2}, 1 \leq i_{3} \leq N_{3}\right\}$. Here,

$$
X_{i j k}= \begin{cases}1 & \text { if the atom located at the point }(i, j, k) \text { is displaced, } \\ 0 & \text { if the atom located at the point }(i, j, k) \text { is not displaced }\end{cases}
$$

for $i=1, \ldots, N_{1}, j=1, \ldots, N_{2}$, and $k=1, \ldots, N_{3}$. It should be noted that in this formulation $N=N_{1} N_{2} N_{3}$ and $N_{1}$ is very large compared to $N_{2}$ and $N_{3}$. Throughout this paper, we use (2).

Let $d_{1}<d_{2}<\cdots<d_{r}$ be the $r$ smallest distances between the points in $D_{N_{1}, N_{2}, N_{3}}$, and define

$$
S\left(N_{1}, N_{2}, N_{3}\right)=\sum_{D_{N_{1}, N_{2}, N_{3}}} X_{i j k}
$$

and

$$
Y_{\ell}\left(N_{1}, N_{2}, N_{3}\right)=\frac{1}{4}\left(\sum_{A_{\ell}\left(N_{1}, N_{2}, N_{3}\right)} X_{i j k} X_{i^{\prime} j^{\prime} k^{\prime}}\right),
$$

where $\ell=1, \ldots, r$ and $A_{\ell}\left(N_{1}, N_{2}, N_{3}\right)$ is the set of all points $(i, j, k) \in D_{N_{1}, N_{2}, N_{3}}$ and $\left(i^{\prime}, j^{\prime}, k^{\prime}\right) \in D_{N_{1}, N_{2}, N_{3}}$ satisfying $d_{\ell}^{2}=\left(i-i^{\prime}\right)^{2}+\left(j-j^{\prime}\right)^{2}+\left(k-k^{\prime}\right)^{2}$.

In order to find the joint distribution of $X_{i j k}$ for $i=1, \ldots, N_{1}, j=1,2, \ldots, N_{2}$, and $k=1, \ldots, N_{3}$, we must first determine how these atoms are linked. Suppose that the atoms 
are identical, i.e. $\mathrm{P}\left(X_{i j k}=1\right)$ does not depend on $i, j$, and $k$, and that the interdependence between the atoms declines with distance.

To capture the above properties, we assume that the joint distribution of $X_{i j k}, i=1, \ldots, N_{1}$, $j=1, \ldots, N_{2}, k=1, \ldots, N_{3}$, is of the form

$$
\mathrm{P}\left(X_{i j k}=x_{i j k} ; i=1, \ldots, N_{1}, j=1, \ldots, N_{2}, k=1, \ldots, N_{3}\right)=C^{-1} \exp \left[\gamma s+\sum_{\ell=1}^{r} \theta_{\ell} y_{\ell}\right],
$$

where $x_{i j k}$ is either 0 or $1, C$ is a normalizing constant, and $s$ and $y_{1}, \ldots, y_{r}$ represent the values of $S\left(N_{1}, N_{2}, N_{3}\right)$ and $Y_{1}\left(N_{1}, N_{2}, N_{3}\right), \ldots, Y_{r}\left(N_{1}, N_{2}, N_{3}\right)$ described in (3) and (4), respectively, when $X_{i j k}=x_{i j k}$ for $i=1, \ldots, N_{1}, j=1, \ldots, N_{2}$, and $k=1, \ldots, N_{3}$. In (5), $\gamma, \theta_{1}, \theta_{2}, \theta_{3}, \ldots, \theta_{r}$ are real-valued parameters. Random variables $X_{i j k}, i=1, \ldots, N_{1}, j=$ $1, \ldots, N_{2}, k=1, \ldots, N_{3}$, having distributions given by (5) are particular examples of Markov random fields. For more properties of (5), we refer the reader to Besag (1974) and Banerjee et al. (2004, pp. 76-84). Throughout this paper, we will use the model (5). We cannot overemphasize the fact that the model entailed in (5) is only one of the many different models that is encountered in practice.

Several remarks are in order with respect to (5). Firstly, note that (5) can be written as

$$
\begin{gathered}
\mathrm{P}\left(X_{i j k}=x_{i j k} ; i=1, \ldots, N_{1}, j=1, \ldots, N_{2}, k=1, \ldots, N_{3}\right) \\
=\left(M\left(\theta_{1}, \theta_{2}, \theta_{3}, \ldots, \theta_{r}\right)\right)^{-1} p^{s} q^{N-s} \exp \left[\sum_{\ell=1}^{r} \theta_{\ell} y_{\ell}\right],
\end{gathered}
$$

where $p=\mathrm{e}^{\gamma} /\left(1+\mathrm{e}^{\gamma}\right), q=1-p$, and $M\left(\theta_{1}, \ldots, \theta_{r}\right)$ is the moment generating function of $Y_{1}\left(N_{1}, N_{2}, N_{3}\right), \ldots, Y_{r}\left(N_{1}, N_{2}, N_{3}\right)$ evaluated at $\theta_{1}, \theta_{2}, \ldots, \theta_{r}$ and computed under the assumption that the $X_{i j k}$ s are independent, identically distributed Bernoulli variables having success parameter $p$; see Saunders et al. (1979) for more details.

Secondly, let

$$
M_{\theta}\left(t_{0}, t_{1}, \ldots, t_{r}\right)=\mathrm{E}\left\{\exp \left[t_{0} S\left(N_{1}, N_{2}, N_{3}\right)+\sum_{i=1}^{r} t_{i} Y_{i}\left(N_{1}, N_{2}, N_{3}\right)\right]\right\}
$$

be the joint moment generating function of

$$
S\left(N_{1}, N_{2}, N_{3}\right), Y_{1}\left(N_{1}, N_{2}, N_{3}\right), \ldots, \quad \text { and } \quad Y_{r}\left(N_{1}, N_{2}, N_{3}\right)
$$

when $\theta=\left(\theta_{1}, \ldots, \theta_{r}\right)$. Then

$$
M_{\theta}\left(t_{0}, t_{1}, \ldots, t_{r}\right)=\frac{M^{*}\left(t_{0}, t_{1}+\theta_{1}, \ldots, t_{r}+\theta_{r}\right)}{M^{*}\left(0, t_{1}, \ldots, t_{r}\right)}
$$

where $M^{*}$ is the joint moment function of

$$
S\left(N_{1}, N_{2}, N_{3}\right), Y_{1}\left(N_{1}, N_{2}, N_{3}\right), \ldots, \quad \text { and } \quad Y_{r}\left(N_{1}, N_{2}, N_{3}\right)
$$

under the assumption that $\theta_{1}=\theta_{2}=\cdots=\theta_{r}=0$. 
Finally, for any $(i, j, k), 1<i<N_{1}, 1<j<N_{2}, 1<k<N_{3}$,

$$
\begin{gathered}
\mathrm{P}\left(X_{i j k}=1 \mid X_{i^{\prime} j^{\prime} k^{\prime}},\left(i^{\prime}, j^{\prime}, k^{\prime}\right) \in T_{\ell}(i, j, k), \ell=1, \ldots, r\right) \\
=\frac{p \exp \left[\sum_{\ell=1}^{r} \theta_{\ell}\left(\sum_{T_{\ell}(i, j, k)} X_{i^{\prime} j^{\prime} k^{\prime}}\right)\right]}{q+p \exp \left[\sum_{\ell=1}^{r} \theta_{\ell}\left(\sum_{T_{\ell}(i, j, k)} X_{i^{\prime} j^{\prime} k^{\prime}}\right)\right]},
\end{gathered}
$$

where $T_{\ell}(i, j, k)=\left\{\left(i^{\prime}, j^{\prime}, k^{\prime}\right):\left(i^{\prime}, j^{\prime}, k^{\prime}\right) \in D_{N_{1}, N_{2}, N_{3}}-\{(i, j, k)\}\right.$ and $\left(i-i^{\prime}\right)^{2}+\left(j-j^{\prime}\right)^{2}+$ $\left.\left(k-k^{\prime}\right)^{2}=d_{\ell}^{2}\right\}$ and, for any two sets $A$ and $B, A-B$ is the complement of $B$ with respect to $A$.

For example, if $i=j=k=2$ and $\ell=1$ then it is clear that $d_{1}=1$,

$$
\begin{aligned}
T_{1}(2,2,2)= & \left\{\left(i^{\prime}, j^{\prime}, k^{\prime}\right):\left(i^{\prime}, j^{\prime}, k^{\prime}\right) \in D_{N_{1}, N_{2}, N_{3}}-\{(2,2,2)\}\right. \text { and } \\
& \left.\left(2-i^{\prime}\right)^{2}+\left(2-j^{\prime}\right)^{2}+\left(2-k^{\prime}\right)^{2}=1\right\} \\
= & \{(2,2,3),(2,3,2),(3,2,2),(2,2,1),(2,1,2),(1,2,2)\},
\end{aligned}
$$

and (8) is

$$
\begin{aligned}
& \mathrm{P}\left(X_{222}=1 \mid X_{i^{\prime} j^{\prime} k^{\prime}},\left(i^{\prime}, j^{\prime}, k^{\prime}\right) \in T_{1}(2,2,2)\right) \\
& \quad=\frac{p \exp \left[\theta_{1}\left(X_{221}+X_{212}+X_{122}+X_{223}+X_{232}+X_{322}\right)\right]}{q+p \exp \left[\theta_{1}\left(X_{221}+X_{212}+X_{122}+X_{223}+X_{232}+X_{322}\right)\right]} .
\end{aligned}
$$

In this paper we focus on two types of linear nanosystems. Both cases describe the relationship between the failure of a linear nanosystem and its atoms.

Type 1. We assume that the system fails if at least $\ell+1$ atoms are displaced. We refer to this type of system as an $\ell$-out-of $N: F$ linear nanosystem.

Type 2. For given integers $r_{1}, r_{2}$, and $r_{3}\left(1 \leq r_{i} \leq N_{i}, i=1,2,3\right)$, let $C_{r_{1}, r_{2}, r_{3}}(i, j, k)=$ $\left\{\left(i_{1}, i_{2}, i_{3}\right): i-r_{1}+1 \leq i_{1} \leq i, j-r_{2}+1 \leq i_{2} \leq j, k \leq i_{3} \leq k+r_{3}-1\right\}$ for $i=$ $r_{1}, \ldots, N_{1}, j=r_{2}, \ldots, N_{2}$, and $k=1, \ldots, N_{3}-r_{3}+1$. Define $W_{i j k}=\sum \sum \sum X_{a b c}$, where the sum is taken over all possible values of $a, b$, and $c$ in $C_{r_{1}, r_{2}, r_{3}}(i, j, k)$. We assume that the system fails if at least one of $W_{i j k}, i=r_{1}, \ldots, N_{1}, j=r_{2}, \ldots, N_{2}, k=1, \ldots, N_{3}-$ $r_{3}+1$, exceeds $\ell$. In this case the system will survive if all the $W_{i j k}, i=r_{1}, \ldots, N_{1}, j=$ $r_{2}, \ldots, N_{2}, k=1, \ldots, N_{3}-r_{3}+1$, are less than or equal to $\ell$.

It should be noted that in practical situations there are different tools, such as scanning probe microscopy, scanning force microscopy, scanning tunneling microscopy, and atomic microscopy, to provide information about $\ell$ for types 1 and 2 linear nanosystems.

This paper is organized as follows. In Section 2 we consider an $\ell$-out-of $N: F$ linear nanosystem (type 1) and assess its limiting reliability when $N$ is assumed to be very large $(N \rightarrow \infty)$. In Section 3 we consider a type 2 linear nanosystem.

\section{Assessing the reliability of an $\ell$-out-of $N: F$ linear nanosystem (type 1)}

Using (3), it is clear that the reliability of an $\ell$-out-of $N: F$ linear nanosystem is

$$
R_{1}(N, \ell)=\mathrm{P}\left(S\left(N_{1}, N_{2}, N_{3}\right) \leq \ell\right) .
$$

That is, the probability of failure is

$$
F_{1}(N, \ell)=1-R_{1}(N, \ell)=\mathrm{P}\left(S\left(N_{1}, N_{2}, N_{3}\right) \geq \ell+1\right) .
$$

Since, for a linear nanosystem, $N$ is usually very large, we will compute $R_{1}(N, \ell)$ when $N \rightarrow \infty$. 
Theorem 1. If $N \rightarrow \infty$ and $\ell \geq 1$,

$$
R_{1}(N, \ell)=\Phi\left(\frac{\ell-N p}{\sqrt{N p(1-p)}}\right)
$$

where $p=\mathrm{e}^{\gamma} /\left(1+\mathrm{e}^{\gamma}\right)$.

Proof. From (7), it is clear that the distribution of $S\left(N_{1}, N_{2}, N_{3}\right)$ for any $\theta_{1}, \ldots$ and $\theta_{r}$ is equivalent to the distribution of $S\left(N_{1}, N_{2}, N_{3}\right)$ under the assumption that $\theta_{1}=\theta_{2}=\cdots=$ $\theta_{r}=0$. Using this result and the central limit theorem, we obtain (9).

As an application of Theorem 1, consider the following example.

Example 1. Suppose that $N=10^{6}$ and that the linear nanosystem fails if at least 1001 atoms are displaced, i.e. 1000-out-of- $10^{6}: F$ linear nanosystem. In (5), suppose that $\gamma=-7$. It is clear that $p \approx 9.2 \times 10^{-4}$. Then, from Theorem 1 ,

$$
R_{1}\left(10^{6}, 1000\right)=\Phi\left(\frac{1000-920}{30.5}\right)=\Phi(2.62)=0.996 .
$$

That is, the limiting reliability of the system with this specification is about 0.996 .

\section{Assessing the reliability of a type 2 linear nanosystem}

It is clear that, for a type 2 linear nanosystem, the reliability is

$$
\begin{aligned}
R_{2}\left(N, r_{1}, r_{2}, r_{3}, \ell\right)= & \mathrm{P}\left(W_{i j k} \leq \ell ; i=r_{1}, \ldots, N_{1}, j=r_{2}, \ldots, N_{2},\right. \\
& \left.\quad k=1, \ldots, N_{3}-r_{3}+1\right) \\
= & \mathrm{P}\left(\max \left(W_{i j k}\right) \leq \ell\right),
\end{aligned}
$$

where the maximum is taken over all points $(i, j, k)$ such that $r_{1} \leq i \leq N_{1}, r_{2} \leq j \leq N_{2}$, and $1 \leq k \leq N_{3}-r_{3}+1$.

To calculate $R_{2}\left(N, r_{1}, r_{2}, r_{3}, \ell\right)$ in (10), instead of working directly with the $W_{i j k} \mathrm{~s}$, we first construct an equivalent parallel sequence of random variables and then use this sequence to obtain $R_{2}\left(N, r_{1}, r_{2}, r_{3}, \ell\right)$. The problem is thus to make sure that both sequences are asymptotically equivalent.

In (5), first we assume that $\theta_{\ell}=0$ and $\ell=1, \ldots, r$. Then

$$
R_{2}\left(N, r_{1}, r_{2}, r_{3}, \ell\right)=\mathrm{P}\left(\max \left(W_{i j k}^{*}\right) \leq \ell\right),
$$

where $W_{i j k}^{*}$ is binomial with parameters $r^{*}=r_{1} r_{2} r_{3}$ and $p$, and the maximum is over all $(i, j, k)$ such that $r_{1} \leq i \leq N_{1}, r_{2} \leq j \leq N_{2}$, and $1 \leq k \leq N_{3}-r_{3}+1$. Below, we calculate (11) for different values of $\ell$. We assume that $N \rightarrow \infty, p \rightarrow 0$ (displacement of an atom is a rare event), and $N p \rightarrow \eta(\eta>0)$.

Case $I: \ell=0$. For this case, it is equivalent to saying that the nanosystem will survive if all its atoms do not move. Thus,

$$
R_{2}\left(N, r_{1}, r_{2}, r_{3}, 0\right)=(1-p)^{N}=\left(1-\frac{\eta}{N}\right)^{N} .
$$

Now, as $N \rightarrow \infty, R_{2}(N, 0)=\mathrm{e}^{-\eta}$. 
Case II: $\ell=1$. Since $X_{i j k}, i=1, \ldots, N_{1}, j=1, \ldots, N_{2}, k=1, \ldots, N_{3}$, are independent, we find that $W_{i j k}^{*}, i=r_{1}, \ldots, N_{1}, j=r_{2}, \ldots, N_{2}, k=1, \ldots, N_{3}-r_{3}+1$, are associated random variables. (For the definition of associated random variables, see Barlow and Proschan (1981, pp. 29-32).) Thus,

$$
\begin{aligned}
& R_{2}\left(N, r_{1}, r_{2}, r_{3}, 1\right)= \mathrm{P}\left(W_{i j k}^{*} \leq 1 ; i=r_{1}, \ldots, N_{1}, j=r_{2}, \ldots, N_{2},\right. \\
&\left.k=1, \ldots, N_{3}-r_{3}+1\right) \\
& \geq \prod_{i=r_{1}}^{N_{1}} \prod_{j=r_{2}}^{N_{2}} \prod_{k=1}^{N_{3}-r_{3}+1} \mathrm{P}\left(W_{i j k}^{*} \leq 1\right) \\
&=\left(r^{*} p(1-p)^{r^{*}-1}+(1-p)^{r^{*}}\right)^{N^{*}},
\end{aligned}
$$

where $N^{*}=\left(N_{1}-r_{1}+1\right)\left(N_{2}-r_{2}+1\right)\left(N_{3}-r_{3}+1\right)$ and $r^{*}=\left(r_{1}\right)\left(r_{2}\right)\left(r_{3}\right)$. Since $N^{*} / N \rightarrow 1,(12)$ reduces to

$$
R_{2}\left(N, r_{1}, r_{2}, r_{3}, 1\right) \geq\left(r^{*} p(1-p)^{r^{*}-1}+(1-p)^{r^{*}}\right)^{N} .
$$

The right-hand side of (13) can be written as

$$
\begin{aligned}
\left(r^{*} p(1-p)^{r^{*}-1}+(1-p)^{r^{*}}\right)^{N} & =\left((1-p)^{r^{*}-1}\left(1-p+r^{*} p\right)\right)^{N} \\
& =\left(\left(1-\frac{\eta}{N}\right)^{r^{*}-1}\left(1-\frac{\eta}{N}+\frac{r^{*} \eta}{N}\right)\right)^{N}
\end{aligned}
$$

It follows that

$$
\lim _{N \rightarrow \infty}\left(\left(1-\frac{\eta}{N}\right)^{r^{*}-1}\left(1-\frac{\eta}{N}+\frac{r^{*} \eta}{N}\right)\right)^{N}=1 .
$$

Consequently, for this case, as $N \rightarrow \infty, R_{2}\left(N, r_{1}, r_{2}, r_{3}, 1\right)=1$.

From case II, it is clear that, for $\theta_{1}=\theta_{2}=\cdots=\theta_{r}=0, R_{2}\left(N, r_{1}, r_{2}, r_{3}, \ell\right)=1$ for $\ell \geq 1$. Keeping the assumptions that $N \rightarrow \infty, p \rightarrow 0$, and $N p \rightarrow \eta(\eta>0)$, and following similar arguments to those in Saunders et al. (1979, p. 558), we can easily show that

$$
\mathrm{P}\left(\max \left(W_{i j k}\right) \leq \ell\right) \approx \mathrm{P}\left(\max \left(W_{i j k}^{*}\right) \leq \ell\right),
$$

where the max is taken over all possible values of $(i, j, k), r_{1} \leq i \leq N_{1}, r_{2} \leq j \leq N_{2}$, $1 \leq k \leq N_{2}-r_{3}+1$. Thus, similar results are obtained for any real numbers $\theta_{1}, \theta_{2}, \ldots, \theta_{r}$. The above results can be summarized in the following theorem.

Theorem 2. If $N \rightarrow \infty, p \rightarrow 0$, and $N p \rightarrow \eta(\eta>0)$, then

(a) $R_{2}\left(N, r_{1}, r_{2}, r_{3}, 0\right)=\mathrm{e}^{-\eta}$,

(b) $R_{2}\left(N, r_{1}, r_{2}, r_{3}, \ell\right)=1$ for $\ell \geq 1$.

Next we move to the case in which $N \rightarrow \infty, p \rightarrow 0$, and $N p^{2} \rightarrow \lambda(\lambda>0)$. To compute $R_{2}\left(N, r_{1}, r_{2}, r_{3}, \ell\right)$, we first prove the following lemma.

Lemma 1. As $N \rightarrow \infty, p \rightarrow 0$, and $N p^{2} \rightarrow \lambda(\lambda>0)$,

$$
\begin{array}{r}
\mathrm{P}\left(X_{i j k}=x_{i j k} ; i=1, \ldots, N_{1}, j=1, \ldots, N_{2}, k=1, \ldots, N_{3}\right) \\
=\prod_{\ell=1}^{r} \exp \left[\lambda_{\ell}-\lambda_{\ell} \exp \left[\theta_{\ell}\right]\right] p^{s}(1-p)^{N-s} \exp \left[\sum_{\ell=1}^{r} \theta_{\ell} y_{\ell}\right],
\end{array}
$$


where $\lambda_{\ell}=\lambda a_{\ell}$ and $4 a_{\ell}$ is the number of $\left(i_{1}, i_{2}, i_{3}\right) \in D_{N_{1}, N_{2}, N_{3}}$ such that $\left(i-i_{1}\right)^{2}+(j-$ $\left.i_{2}\right)^{2}+\left(k-i_{3}\right)^{2}=d_{\ell}^{2}$ for a given $(i, j, k)$. Here $1<i<N_{1}, 1<j<N_{2}$, and $1<k<N_{3}$.

Proof. Using similar arguments to the proof of Theorem 2 of Saunders et al. (1979), we can show that $Y_{1}\left(N_{1}, N_{2}, N_{3}\right), \ldots, Y_{r}\left(N_{1}, N_{2}, N_{3}\right)$ are independent and that $Y_{\ell}\left(N_{1}, N_{2}, N_{3}\right)$ is a Poisson random variable with mean $\lambda a_{\ell}, \ell=1, \ldots, r$. Now, from (6) and the fact that, for a Poisson distribution with mean $\mu$, the moment generating function is $\exp \left[-\mu+\mu \mathrm{e}^{t}\right]$, we obtain the result.

If $\ell=0$ then, using Theorem 1 and Theorem 2 of Saunders et al. (1979),

$$
R_{2}\left(N, r_{1}, r_{2}, r_{3}, 0\right)=\mathrm{P}\left(S\left(N_{1}, N_{2}, N_{2}\right)=0\right) \approx \Phi\left(\frac{(1 / 2-N p)}{\sqrt{N p(1-p)}}\right) \approx 0 .
$$

Now consider the case in which $\ell>0, N \rightarrow \infty, p \rightarrow 0, N p^{2} \rightarrow \lambda(\lambda>0)$, and $\theta_{1}=\cdots=\theta_{r}=0$. For this case,

$$
R_{2}\left(N, r_{1}, r_{2}, r_{3}, \ell\right)=\mathrm{P}\left(W_{i j k}^{*} \leq \ell ; i=r_{1}, \ldots, N_{1}, j=r_{2}, \ldots, N_{2}, k=1, \ldots, N_{3}-r_{3}+1\right) .
$$

First note that, for any $(i, j, k)$, for $\ell=1$ and $N \rightarrow \infty$,

$$
\begin{aligned}
\mathrm{P}\left(W_{i j k}^{*} \leq 1\right) & =\lim _{N \rightarrow \infty}\left((1-p)^{r^{*}}+p r^{*}(1-p)^{r^{*}-1}\right) \\
& =\lim _{N \rightarrow \infty}\left(\left(1-\frac{\sqrt{\lambda}}{\sqrt{N}}\right)^{r^{*}}+r^{*}\left(\frac{\sqrt{\lambda}}{\sqrt{N}}\right)\left(1-\frac{\sqrt{\lambda}}{\sqrt{N}}\right)^{r^{*}-1}\right) \\
& =1 .
\end{aligned}
$$

Again, since the $W_{i j k}^{*}$ s are associated, we obtain

$$
\mathrm{P}\left(\max \left(W_{i j k}^{*}\right) \leq 1\right)=1 .
$$

That is, as $N \rightarrow \infty, R_{2}\left(N, r_{1}, r_{2}, r_{3}, 1\right)=1$. Thus, as $N \rightarrow \infty, R_{2}\left(N, r_{1}, r_{2}, r_{3}, \ell\right)=1$ for $\ell \geq 1$.

It should be noted that, from (8), if $\theta_{1}, \theta_{2}, \ldots, \theta_{r} \geq 0$ then the $X_{i j k}$ s are conditionally increasing in sequence and, therefore, they are associated random variables. For the definition of conditionally increasing in sequence, see Barlow and Proschan (1981, pp. 146-148). Using this fact and similar arguments to Theorem 2, the $W_{i j k}$ s are also associated random variables and, thus, for this case, $R_{2}\left(N, r_{1}, r_{2}, r_{3}, \ell\right)=1$ as well.

Below we summarize the above results.

Theorem 3. Suppose that $N \rightarrow \infty, p \rightarrow 0$, and $N p^{2} \rightarrow \lambda(\lambda>0)$. Then

(a) $R_{2}\left(N, r_{1}, r_{2}, r_{3}, 0\right)=0$,

(b) if $\theta_{i} \geq 0, i=1,2, \ldots, r, R_{2}\left(N, r_{1}, r_{2}, r_{3}, \ell\right)=1, \ell \geq 1$.

For other cases, it is difficult to directly assess the reliability $R_{2}\left(N, r_{1}, r_{2}, r_{3}, \ell\right)$. We obtain it through simulations that are carried out in two stages. It should be noted that the technique we describe below can also be used for small $N$ as well.

Stage 1. At this stage we compute $\lambda_{i j k}=\mathrm{P}\left(W_{i j k}>\ell, i=r_{1}, \ldots, N_{1}, j=r_{2}, \ldots, N_{2}\right.$, $\left.k=1, \ldots, N_{3}-r_{3}+1\right)$. There are many situations where it is hard to compute the $\lambda_{i j k} \mathrm{~s}$ analytically. For those situations, using conditional probabilities in (8) for a given $(i, j, k), r_{1} \leq$ $i \leq N_{1}, r_{2} \leq j \leq N_{2}, 1 \leq k \leq N_{3}-r_{3}+1$, and techniques like the Gibbs sampler, we can 
generate $W_{i j k}$ and then use it to estimate $\lambda_{i j k}=\mathrm{P}\left(W_{i j k}>\ell\right)$. For more details about the Gibbs sampler, see Casella and George (1992).

Stage 2. Suppose that

$$
\lambda=\sum_{i} \sum_{j} \sum_{k} \lambda_{i j k} \quad \text { and } \quad V_{i j k}= \begin{cases}1 & \text { if } W_{i j k}>\ell, \\ 0 & \text { otherwise }\end{cases}
$$

Then it is clear that $R_{2}\left(N, r_{1}, r_{2}, r_{3}, \ell\right)=1-\mathrm{P}(V>0)$, where

$$
V=\sum_{i=r_{1}}^{N_{1}} \sum_{j=r_{2}}^{N_{2}} \sum_{k=1}^{N_{3}-r_{3}+1} V_{i j k}
$$

Here $V$ represents the total number of $W_{i j k}$ s that exceed $\ell$.

Now, let $I$ be a random variable independent of the random vector

$$
\left(V_{r_{1} r_{2} 1}, \ldots, V_{N_{1} N_{2}\left(N_{3}-r_{3}+1\right)}\right)
$$

and defined on $\left\{(i, j, k) ; r_{1} \leq i \leq N_{1}, r_{2} \leq j \leq N_{2}, 1 \leq k \leq N_{3}-r_{3}+1\right\}$ such that

$$
\mathrm{P}(I=(i, j, k))=\frac{1}{\left(N_{1}-r_{1}+1\right)\left(N_{2}-r_{2}+1\right)\left(N_{3}-r_{3}+1\right)} .
$$

Then,

$$
\mathrm{P}\left(I=(i, j, k) \mid V_{I}=1\right)=\frac{\lambda_{i j k}}{\lambda}
$$

Let

$$
R= \begin{cases}0, & V=0 \\ \frac{1}{V}, & V>0\end{cases}
$$

Then

$$
\begin{aligned}
\mathrm{P}(V>0) & =\mathrm{E}\{R V\} \\
& =\sum_{i=r_{1}}^{N_{1}} \sum_{j=r_{2}}^{N_{2}} \sum_{k=1}^{N_{3}-r_{3}+1} \mathrm{E}\left\{R V_{i j k}\right\} \\
& =\sum_{i=r_{1}}^{N_{1}} \sum_{j=r_{2}}^{N_{2}} \sum_{k=1}^{N_{3}-r_{3}+1} \mathrm{E}\left\{R V_{i j k} \mid V_{i j k}=1\right\} \lambda_{i j k} \\
& =\sum_{i=r_{1}}^{N_{1}} \sum_{j=r_{2}}^{N_{2}} \sum_{k=1}^{N_{3}-r_{3}+1} \lambda_{i j k} \mathrm{E}\left\{R \mid V_{i j k}=1\right\} .
\end{aligned}
$$

Using the fact that

$$
\begin{aligned}
\mathrm{E}\left\{R \mid V_{I}=1\right\} & =\sum_{i=r_{1}}^{N_{1}} \sum_{j=r_{2}}^{N_{2}} \sum_{k=1}^{N_{3}-r_{3}+1} \mathrm{E}\left\{R \mid I=(i, j, k), V_{I}=1\right\} \mathrm{P}\left(I=(i, j, k) \mid V_{I}=1\right) \\
& =\sum_{i=r_{1}}^{N_{1}} \sum_{j=r_{2}}^{N_{2}} \sum_{k=1}^{N_{3}-r_{3}+1} \frac{\lambda_{i j k}}{\lambda} \mathrm{E}\left\{R \mid V_{i j k}=1\right\}
\end{aligned}
$$


and (14),

$$
\mathrm{P}(V>0)=\lambda \mathrm{E}\left\{\frac{1}{V} \mid V_{I}=1\right\} .
$$

At this stage, we simulate the value of $J$, a random variable with probability function

$$
\mathrm{P}(J=(i, j, k))=\frac{\lambda_{i j k}}{\lambda}
$$

for $i=r_{1}, \ldots, N_{1}, j=r_{2}, \ldots, N_{2}$, and $k=1, \ldots, N_{3}-r_{3}+1$. Note that the random variable $J$ has the same distribution as $\mathrm{P}\left(I=(i, j, k) \mid V_{I}=1\right)$. Now, we set $V_{i j k}=1$ for $J=(i, j, k)$, generate $W_{i^{\prime} j^{\prime} k^{\prime}}$ for $r_{1} \leq i^{\prime} \leq N_{1}$ and $r_{2} \leq j^{\prime} \leq N_{2}, 1 \leq k^{\prime} \leq N_{3}-r_{3}+1$ such that $\left(i^{\prime}, j^{\prime}, k^{\prime}\right)$ is not equal to $J$, and compute $V_{i^{\prime} j^{\prime} k^{\prime}}$ and, consequently,

$$
V^{*}=\sum_{i=r_{1}}^{N_{1}} \sum_{j=r_{2}}^{N_{2}} \sum_{k=1}^{N_{3}-r_{3}+1} V_{i j k} .
$$

It should be noted that $V^{*} \geq 1$. Now, we estimate $R_{2}\left(N, r_{1}, r_{2}, r_{3}, \ell\right)=1-\mathrm{P}(V>0)$ by $1-\lambda\left(1 / V^{*}\right)$.

\section{Acknowledgements}

The author wishes to thank the anonymous referee for some important remarks about improvements both in the form and in the content.

\section{References}

Bahar, R. I., TAhoori, M. B., Shukla, S. And Lombardi, F. (2005). Special Issue on 'Challenges for Reliable design at Nanoscale'. IEEE Design and Test, July-August 2005.

Banerjee, S., Carlin, B. And Gelfand, A. E. (2004). Hierarchical Modeling and Analysis for Spatial Data. Chapman and Hall, New York.

Barlow, E. And Proschan, F. (1981). Statistical Theory of Reliability and Life Testing: Probability Models. To begin with, Silver Spring, MD.

BESAG, J. (1974). Spatial interaction and the statistical analysis of lattice systems. J. R. Statist. Soc. Ser. B 36, $192-236$.

Bhushan, B. (2007). Springer Handbook of Nano-Technology, 2nd edn. Springer, Berlin.

Casella, G. and George, E. I. (1992). Explaining the Gibbs sampler. Amer. Statist. Assoc. 46, 167-174.

DreXler, K. E. (1992). Nanosystems: Molecular, Machinery, Manufacturing and Computation. John Wiley, New York.

Ebrahimi, N. (1990). Binary structure functions with dependent components. Adv. Appl. Prob. 22, 627-640.

NATVIG, B. (2007). Multistate reliability theory. In Encyclopedia in Quality and Reliability, eds F. Ruggeri et al., John Wiley, New York, pp. 1002-1010.

Poole, C. P. And Owens, F. J. (2003). Introduction to Nanotechnology. John Wiley, New York.

Ratner, M. AND Ratner, D. (2003). Nanotechnology: A Gentle Introduction to the Big Idea. Prentice Hall, Englewood Cliffs, NJ.

SAUNDERS, R., KRYSCIO, R. AND FUnK, G. (1979). Limiting results for arrays of binary random variables on rectangular lattices under sparseness conditions. J. Appl. Prob. 16, 554-566.

Tabata, O. And Tsuchiya, T. (2008). Reliability of MEMS. John Wiley, New York. 